\title{
Vom Inkubationsritus zur Psychotherapie
}

\section{Eine transkulturelle Perspektive}

\author{
Tania Re \\ Psychotherapie-Wissenschaft 9 (1) 53-59 2019 \\ www.psychotherapie-wissenschaft.info \\ CC BY-NC-ND \\ https://doi.org/10.30820/1664-9583-2019-1-53
}

\begin{abstract}
Zusammenfassung: Ursprünglich, im Westen, war die Medizin, die die Pflege von Körper, Geist und Seele betraf, eine divinatorische Kunst. Im Laufe der Zeit hat der Begriff «Weissagung» seine ursprüngliche Bedeutung verloren, im Vergleich zur Antike und insbesondere zur archaischen griechischen Welt, wo er einen enormen Wert hatte. Die Kunst des Asklepios (Äskulap) ist, wie die seines Vaters Apollo, eine göttliche Kunst, die ein Heilritual voraussetzt. In diesem Zusammenhang sei daran erinnert, dass die historisch-anthropologische Forschung in vielen Kulturen des schamanischen Typs (Südamerika, Sibirien, Indien) im therapeutischen Bereich eine sehr alte Verwendung von «Meisterpflanzen» oder Pflanzen aufzeichnet, die es aufgrund ihrer Eigenschaften ermöglichen, «aussergewöhnliche Bewusstseinszustände» zu erreichen. Diese Bewusstseinszustände können durch verschiedene Praktiken wie Meditation, holotropes Atmen, Yoga, sensorische Entbehrung oder sogar spontan wie bei den christlichen Mystikern entstehen. Die von den Forschern beschriebenen aktuellen Erfahrungen liefern uns sehr ermutigende Daten über den Nutzen dieser Art von Erkenntnissen. Die in den USA und in Europa, in Spanien und der Schweiz entwickelte Forschung folgt tendenziell Forschungsprotokollen, in denen das Set (der aktuelle Stand, aber auch die klinische und biographische Geschichte des Patienten), das Setting (das Umfeld, in dem die Erfahrung stattfindet) und die Substanz (Art, Dosierung, Qualität und Quantität) eine grundlegende Rolle spielen. Die Herausforderungen für die Zukunft im psychotherapeutischen Bereich über den therapeutischen Einsatz psychoaktiver Substanzen sind nach wie vor vielfältig, eine echte Möglichkeit könnte darin bestehen, den rituellen Einsatz von Substanzen durch die Kombination von Vergangenheit und Gegenwart, altem Wissen und moderner Wissenschaft im Dienste der Pflege zu revitalisieren.
\end{abstract}

Schlüsselwörter: Asklepios (Äskulap), Epidauros, sapientale Medizin («Weisheitsmedizin»), Tempelmedizin, Inkubationsheiligtum, «Meisterpflanzen», Schamanismus

\section{Die Ursprünge: Die Medizin im Asklepieion}

Ursprünglich, im Westen, war die Medizin, die die Pflege von Körper, Geist und Seele betraf, eine divinatorische Kunst. Im Laufe der Zeit hat der Begriff «Weissagung» seine ursprüngliche Bedeutung verloren, im Vergleich zur Antike und insbesondere zur archaischen griechischen Welt, wo sie einen enormen Wert hatte. Die Weissagung war in der Tat die reinste Form der Erkenntnis, eine Art halbgeschlossene Tür oder Brücke, die die menschliche Welt mit der göttlichen Welt verband. Epidaurus war in der Antike eine «therapeutische Landschaft», ein architektonischer Komplex, der mehrere Gebäude umfasste, zu dem Menschen aus ganz Griechenland kamen, um vom Gott Asklepios, einer seit 500 v. Chr. verehrten Gottheit, geheilt zu werden. Epidaurus war eine sehr komplexe Struktur, eine Art Stadtstaat oder souveräner Staat, der Tausende von Anhängern aufnehmen konnte.

Die emblematische und schillernde Figur Asklepios' gilt auch heute noch als Gott der Medizin. Er ist mit einer medizinischen Anschauung verbunden, die als «Tempelmedizin» definiert wurde, zu deren bedeutendstem Zentrum Epidauros wurde.

Man muss sich zunächst eine gewisse Konkurrenz zwischen den verschiedenen Städten der Asklepieia vor- stellen, ein Wettbewerb, der später dank der Antwort des Delphischen Orakels beigelegt wurde.

Letztere erkannte die herausragende Rolle des Heiligtums von Epidauros über alle anderen; es ist Pausanias (II, 27, 6), der uns erzählt, wie das Orakel von Delphi auf die Frage des Apollophanes nach Asklepios' Herkunft antwortete: «O Asklepios, geboren zum Heil aller Sterblichen, die mit mir [Apollo] in Liebe vereinte Tochter von Phlegia, die reizende Koronis, gebar dich im steinigen Epidauros».

Das Asklepieion befand sich in einer flachen, wasserreichen Gegend am Fusse des Berges Kynortion; die Gläubigen erreichten das Heiligtum entlang einer Strasse aus Argos oder aus Epidauros selbst. In der Stadt war der Asklepios-Kult seit dem 6. Jahrhundert v. Chr. fest etabliert und erreichte im 4. Jahrhundert die volle Hegemonie, zu Lebzeiten des Hippokrates. Das ummauerte Heiligtum stand ausserhalb der Stadt und war mit dieser durch eine von Statuen flankierte Strasse verbunden.

Der einführende Torbau, als Propylon bezeichnet, war mit Säulen geschmückt und markierte den Eingang zum Heiligtum; hier erinnern die literarischen Quellen an das Vorhandensein einer grossen Inschrift, die schon von weitem sichtbar war und die Gläubigen beim Erreichen des Heiligtums ermahnte: "Rein muss sein, wer den duf- 
tenden Tempel betritt; aber rein zu sein bedeutet, heilige Gedanken zu haben» (Porphyrios, De abst., II, 19).

Nach dem Eingang erlaubte ein Brunnen aus dem 6. Jahrhundert v. Chr. den Pilgern, sich nach der langen Reise zu waschen und zu erfrischen; er bot auch das Wasser für die Opferrituale. Der Tempel selbst, Werk des Architekten Theodotos, bestand aus Kalkstein. Die Zelle in der Mitte war von dorischen Säulen umgeben und mit polychromem Marmor verziert; sie beherbergte die chryselephantine (mit Gold und Elfenbein bearbeitete) Statue des Asklepios.

Asklepios wird als reifer Mann dargestellt, mit Bart (aber anderenorts auch als junger Mann ohne Bart), auf dem Thron sitzend, mit einem Stock in der Faust, während die andere Hand auf dem Kopf einer Schlange ruht; zu seinen Füssen liegt ein Hund. Hunde und Schlangen, zusammen mit den weisen Eulen und ernährenden Ziegen, waren heilige Tiere: Ihre Zunge galt als wirkungsvoll durch Lecken, Beruhigen und Heilen von Wunden. In den Säulengängen vor dem Tempel befand sich das Abaton, Aufenthaltsort für die Kranken und Schlafstätte, wo die Kranken die Nächte in der «Inkubation» (Tempelschlaf) verbrachten.

\section{Der Ritus der Trauminkubation (Enkoimesis)}

Der Ritus der Incubatio ist, wie wir sehen werden, die Praxis derer, die schlafen, um Antworten zu erhalten. Der Kranke «incubante ad Asclepio» ist derjenige, der im Asklepieion schläft, um wundersam geheilt aus dem Schlaf aufzuwachen oder auf untergeordnete Weise ein Gebot oder einen Ratschlag zu empfangen, wie er sich zur Heilung verhalten sollte.

In der heiligen Klausur befand sich auch die Tholos, ein kreisförmiges Labyrinth, in dem sich der heilige Brunnen befand, Sitz der heiligen Schlangen, an dem die Kranken die Votivtafeln zurückliessen, auf denen sie ihre Geschichten, mit den aufgetretenen Symptomen und den erhaltenen Behandlungen geschrieben hatten. Ausserhalb der Anlage wurde das monumentale Ensemble durch die chronologisch späteren Gebäudeanbauten der Turnhalle für körperliche Übungen, der Bäder und des Odéion für musikalische Darbietungen zu therapeutischen Zwecken ergänzt.

Das Katagòghion, ein Gästehaus mit mehreren Zimmern, die unter den Arkaden und um die Innenhöfe herum angeordnet waren, bot denjenigen Unterkunft, die auf Wallfahrt zum Heiligtum kamen, um Heilung zu suchen. Die Pilger wurden von einem Empfangspersonal begrüsst, das sich aus Trägern, Verwahrer der Schlüssel zum Tempel und den Schriftgelehrten zusammensetzte, die die Opfergaben jedes Pilgers aufzeichneten und die wirtschaftlichen Verhältnisse berücksichtigten. Der Tempel war jedermann zugänglich, aber den Reichen wurden zusätzliche Gebühren, sogenannte iatras, auferlegt. Iatrós war in der Tat einer der Namen von Asklepios, der auch «König», «Held», «Dämon», «Despot» und «Gott» genannt wurde.

Das Hilfspersonal war vielfältig: Es reichte von Betreuern, die über die Schläfer wachten, über Pyrophoren, die für Beleuchtung sorgten, bis hin zu den Asklepiaden, die die Opfer beaufsichtigten, den Erzählungen der Träume lauschten und die Antworten des Heilgottes übermittelten. An der Spitze stand der Hierophant, der Hohepriester und «Enthüller der heiligen Geheimnisse», der grösste Vertreter spiritueller Macht und Weisheit im Tempel.

Das Zugangsritual bestand in der vorbereitenden Reinigung der Kranken, mit Waschen und kurzem Fasten, gefolgt vom Opfer: «ein Hahn an Asklepios», wie Platon Sokrates in Phaidon in seiner letzten Rede vor seinen Freunden sagen liess. Im Asklepios wurden auch andere Tiere geopfert, und je mehr man hatte, desto mehr gab man.

Nach dem Opfer, stets begleitet von Anrufung, einem ständigen Element dieses Rituals, legte sich der Patient auf ein Lager, das manchmal aus den Häuten der geopferten Tiere bestand: eine Ziege, ein Ochse, ein Hausrind. Dann schlief er ein und träumte; manchmal wachte er wundersam geheilt auf; manchmal erwachte er und war bereit, sich dem Asklepiaden anzuvertrauen, um therapeutische Anweisungen von ihm zu erhalten. Die Therapie der Heilung beruhte ganzheitlich auf einem Regime, das der Gott dem Patienten vorschrieb, das heisst auf Ernährungsregeln, Übungen, Bädern, "die teils seinen Leiden folgen, teils Krankheiten vorbeugen».

Wie man feststellen kann, hat die Therapie nichts Wunderbares oder Göttliches an sich, sondern basiert auf einer Reihe von Verhaltensweisen, die dazu dienen sollen, das körperliche Gleichgewicht wiederherzustellen, das durch die verschiedenen Leiden beeinträchtigt wurde. Die Kunst des Asklepios ist, wie die seines Vaters Apollo, eine göttliche Kunst, die ein Heilritual voraussetzt. Aber sobald wir den Höhepunkt solchen Wissens berücksichtigen, trägt es bereits den Samen seiner Dekadenz in sich. Die Geburt der so genannten technischen Medizin wird die fortschreitende, ich würde sogar unterstreichen, initiatorische Loslösung von dieser Form des Wissens sanktionieren.

Technik - téchne - ist in der Tat ein homerisches Hauptwort, ein altgriechischer Begriff ionischer Herkunft, der eine Kunst, ein Handwerk, einen Beruf bezeichnet. Historisch entwickelte sich der Ausdruck im geografischen Gebiet des Ionischen Meers und der Ägäis, zwischen Magna Graecia und griechischem Mutterland sowie zwischen diesem und Kleinasien, im 5. Jahrhundert v. Chr.: eine Kunst im medizinischen Bereich, verbunden mit dem Namen Hippokrates. Der Begriff Téchne umfasst mehrere Bedeutungen. Es bezeichnet den Beruf des Handwerkers, des Geometers, der die Erde vermisst, des Mathematikers, der die Theoreme erstellt, des Musikers, der Harmonien findet und erzeugt, des Arztes, der die Gesundheit der Menschen pflegt und wiederherstellt. Es handelt sich in jedem Fall um einen Beruf, der Theorie und Praxis in unterschiedlichem Masse beinhaltet und der den Einsatz von intellektuellen Prozessen erfordert.

Im Falle des Arztes, der die Pflegekunst mit Intellekt und Hand ausübt, beziehen sich die Verfahren dieser Wissensmacht einerseits auf die erarbeiteten Formen des geistigen Wissens, andererseits auf Praktiken mit einem konsistenten Inhalt von manuellen Fähigkeiten. Nicht zuletzt unter den Techniken, ja vor der Bedeutung ihres 
Gegenstandes, ist die Medizin eine Tätigkeit der menschlichen Vernunft, die zwischen intellektueller Kenntnis und konkretem manuellem Handeln stattfindet.

Praxis und Logos werden das Wissen von Asklepios zunehmend ersetzen. Diese Vision, in der die Entwicklung der Medizin als Technik als eine Art Abstammung von dieser Tempelmedizin über eine fortschreitende «Entsakralisierung» angesehen wird, geht historisch auf den positivistischen Philosophen Littré zurück, der berühmte Verleger der Werke des Hippokrates im 19. Jahrhundert.

\section{Die Geburt der Hippokratischen Medizin}

Iatrèia, die Kunst der Heilung unter Einsatz von Heilmitteln, erwirbt ihre Physiognomie und Autonomie durch eine Technik, die mit Hippokrates reifte.

Hippokrates, der unbestrittene Vater der modernen Medizin, wird eine fast hundertjährige Langlebigkeit zugeschrieben, die mehr als die Hälfte des 5. und einen Grossteil des 4. Jahrhunderts v. Chr. umfasst. Von seinem ersten Biographen Soranos, Autor des Werks Genealogie und Leben des Hippokrates, wird der «alte Mann von Kos» als Nachkomme des Asklepios der 19. Generation mythisiert, um eine Kontinuität zwischen Asklepiaden und «Technikern» der Gesundheit zu symbolisieren.

Trotz dieser göttlichen Abstammung können wir nicht umhin, an Hippokrates zu denken als ersten «Rationalisten», als Urheber der Entmystifizierung der magischen Aura, die bis zu seinem Erscheinen die Heilungsrituale umgab. In diesem Zusammenhang erscheint es angebracht, seine eigenen Worte über die «alte Medizin» zu zitieren:

«Seit langem verfügt die Medizin über alle Elemente, und das Prinzip und der Weg wurden entdeckt, dank derer über einen langen Zeitraum viele ausgezeichnete Entdeckungen gemacht wurden, und der Rest wird in der Zukunft entdeckt werden, wenn jemand, der dazu fähig ist und sich des bereits Entdeckten bewusst ist, von diesem Ausgangspunkt aus die Forschung weiterführen wird. Wer all dies ablehnt und auf einem anderen Weg und nach einem anderen Schema forscht und behauptet, etwas entdeckt zu haben, hat sich getäuscht und täuscht sich, weil es unmöglich ist. Und aus welchen zwingenden Gründen es unmöglich ist, werde ich versuchen, zu beweisen, indem ich erkläre und demonstriere, was Kunst ist» (Hippokrates, Werke).

Auch Hippokrates, den wir als Gründervater der technischen Medizin definiert haben, spricht von Kunst. Am Anfang bestand zwischen den beiden Figuren, Hippokrates einerseits und Asklepios andererseits, wenn wir einen etwas seltsamen Ausdruck verwenden wollen, eine «stillschweigende Vereinbarung»: Sie lebten in der Tat noch lange zusammen weiter.

Die Auseinandersetzung mit dem Mythos von Asklepios könnte hier enden, aber man darf einen weiteren Umstand nicht vergessen: Der Evangelist Johannes $(4,42)$ berichtet, dass Aesculapius (die lateinische Übersetzung von Asklepios), der als Retter verehrt wurde, lange Zeit ein gefürchteter Konkurrent Jesu Christi war und der Kult dieses Gottes rivalisierte lange Zeit mit dem Christentum. Aus diesem Grund fielen die mit dem Asklepios-Kult verbundenen Anlagen der späteren christlichen Zerstörung zum Opfer.

\section{Von der westlichen Medizin zu den "anderen" traditionellen medizinischen Praktiken}

Im therapeutischen Bereich zeichnet die historischanthropologische Forschung in vielen schamanischen Kulturen (Südamerika, Sibirien, Indien) eine sehr alte Verwendung von «Meisterpflanzen» auf, oder von Pflanzen, die aufgrund ihrer Eigenschaften «ungewöhnliche Bewusstseinszustände» hervorrufen können. Diese Bewusstseinszustände können durch verschiedene Praktiken wie Meditation, holotropes Atmen, Yoga, sensorische Entbehrung oder sogar spontan wie bei den christlichen Mystikern entstehen.

Historisch gesehen lässt sich feststellen, dass in Europa, zuerst zur Zeit der Griechen und dann während des Römischen Reiches, die dionysischen Riten, die Mysterien von Eleusis praktiziert wurden, bei denen auch der Konsum psychoaktiver Substanzen überliefert ist. Ein typisches Element des Dionysos-Kultes ist die im Wesentlichen weibliche Teilnahme an den Zeremonien, die in verschiedenen Teilen Griechenlands gefeiert wurden: Die Bakchen (auch Mänaden genannt) beschworen und besangen ihre Anwesenheit und reproduzierten, auch mittels Masken (wichtig im Dionysos-Kult, der angeblich mit der Geburt der griechischen Tragödie verbunden ist), rituell die mythische Dionysos-Prozession von Silen, Satyre und Nymphen.

Wie McKenna (1991), einer der bedeutendsten Erforscher des schamanischen Phänomens, uns daran erinnert:

«Der Schamanismus ist eine experimentelle Wissenschaft, die sich mit einem Bereich beschäftigt, von dem wir nichts wissen. Es ist wichtig, sich daran zu erinnern, dass sich unsere erkenntnistheoretischen Werkzeuge im Westen sehr ungleich entwickelt haben.

Wir wissen viel über das, was im Herzen des Atoms passiert, aber absolut nichts über die Natur des Geistes. Wir haben keine Hinweise. Wenn die mathematische Formulierung die Grundlage ideologischer Gewissheit sein soll, dann haben wir im Bereich dessen, was wir den Geist nennen, gar keine Gewissheit. Unbewusst gehen wir von allen möglichen Dingen aus, aber wenn wir mit dem Rücken zur Wand stehen, sind wir nicht in der Lage, unsere Position zu verteidigen. Ich glaube, dass Folgendes geschehen ist: Aufgrund der Psychedelik einerseits und der Quantenphysik andererseits wurde das Programm des rationalen Verständnisses der Natur so weit vorangetrieben, bis wir dadurch das irrationale Herz der Natur selbst erreichten. In den Heilpraktiken der prä-hippokratischen Zivilisationen, die wir heute als Magie bezeichnen, gehörten Religion und Fürsorge einer 
einzigen Welt an und galten alle als Ausdruck übernatürlicher Kräfte. Krankheit und Heilung wurden daher als das Ergebnis übernatürlicher Eingriffe angesehen.»

\section{Die «Meisterpflanzen» in der traditionellen Medizin: vom Wald zum Labor}

Heute wie damals berichtet die Forschung über Veränderungen im prosozialen Verhalten in zahlreichen untersuchten Menschengruppen, die verschiedene Arten von «Meisterpflanzen» konsumiert haben, sowohl in religiösen Kontexten, wie Ayahuasca-Sud für Praktizierende der Santo-Daime-Kirche, als auch in informellen Kontexten.

$\mathrm{Zu}$ den Ayahuasca-haltigen Pflanzen gehören Banisteripsis caapi und Psychotria viridis; letztere enthält hohe Dosen von N, N-Dimethyltriptamin, bekannt als DMT, das vom menschlichen Gehirn produziert und in vielen pflanzlichen Substanzen enthalten ist. Die ersten wissenschaftlichen Studien über die psychotropen Wirkungen dieser Substanz begannen Mitte der 1950er Jahre und wurden vom ungarischen Psychiater und Chemiker Stephen Szára, geboren 1923, durchgeführt. Er hat als erster die entheogenen Eigenschaften von DMT entdeckt und erkannt, indem er die Substanz an sich selbst experimentierte.

Doch es war Rick Strassmann, Psychiater und klinischer Psychopharmakologe, geboren 1952, der das Verdienst hatte, das akademische Interesse an der wissenschaftlichen Erforschung psychoaktiver Substanzen zu wecken; Strassman definierte DMT als «das Molekül des Geistes», was auf die tief psychisch-geistigen Auswirkungen im Zusammenhang mit dieser Substanz hindeutet (Bragazzi et al., 2018a-d).

Nach einer Reihe vertiefter Untersuchungen zu den Wirkungen durch Verabreichung von DMT kam Strassman zu einer wichtigen Schlussfolgerung. Die veränderten Bewusstseinszustände, die durch diese Substanz induziert werden - DMT kommt in vielen Pflanzen sowohl in Europa als auch insbesondere in Südamerika vor - können mit mystischen Erfahrungen verglichen werden:

\footnotetext{
«Diese Substanz kann uns zur Annahme der Koexistenz von Gegensätzen wie Leben und Tod, Gut und Böse führen; die Erkenntnis, dass das Bewusstsein auch nach dem Tod fortbesteht; ein tiefes Verständnis der grundlegenden Einheit aller Phänomene; und ein Gefühl der Weisheit oder Liebe, das die ganze Existenz durchdringt» (Strassman, 2001).
}

Strassman stellte zudem fest, dass Nahtod-Erfahrungen (NTE) mit DMT-Erfahrung verglichen werden können; tatsächlich berichteten viele der Teilnehmer an seinen DMT-Studien über Kontakte zu fühlenden und körperlosen Wesen während der Erfahrung.

Aber welches sind die Eigenschaften dieses wirkungsvollen Moleküls? Laut Strassman (2001) kann DMT als das einfachste der psychoaktiven Tryptamine angesehen werden. Seine Pharmakologie wirkt auf Serotonin-
Rezeptorstellen genauso wie andere Substanzen wie Lysergsäurediethylamid (LSD), Psilocybin und Meskalin. Serotonin-Rezeptoren sind im gesamten menschlichen Körper verbreitet und kommen in verschiedenen Geweben und Organen vor, darunter in Blutgefässen, Muskeln, Drüsen und der Haut.

Der Nobelpreisträger Julius Axelrod (1912-2004) berichtete 1972, dass er DMT im menschlichen Hirngewebe isoliert habe, und andere Forschungen haben gezeigt, dass DMT auch in der Zerebrospinalflüssigkeit, dem sogenannten Gehirnwasser, zu finden ist.

Die Zirbeldrüse wird oft als die DMT-Fabrik des menschlichen Körpers angesehen, und Strassman hat die Kenntnis verbreitet, dass das Gehirn beim Träumen und in der Phase vor dem Tod grosse Mengen dieser Verbindung freisetzt. Laut Strassman haben Gewebe und Organe wie Lunge, Leber, Blut und Augen zwar alle Enzyme, die benötigt werden, um Tryptamin in DMT umzuwandeln, aber die Zirbeldrüse ist besonders reich an dieser Substanz; Strassman spekulierte auch, dass die Zirbeldrüse nach dem Tod noch einige Stunden lang postmortales DMT produzieren könne.

Dr. Ede Frecska, Präsident des Psychiatrie-Instituts an der Medizinischen Fakultät der Universität Debrecen, Ungarn, hat kürzlich entdeckt, dass sich DMT an den Sigma-1-Rezeptor $(\sigma 1 R)$ binden kann. Es handelt sich um ein transmembranes Protein, das im ganzen Körper und vor allem in einigen Regionen des zentralen Nervensystems vorhanden ist. Dieser Rezeptor spielt eine Schlüsselrolle beim Schutz der Zellen vor Apoptose bei kritisch niedriger Sauerstoffkonzentration (d.h. unter hypoxischen Bedingungen) und lässt Raum für das faszinierende Argument, dass DMT tatsächlich während des Todes in grossen Mengen produziert und freigesetzt werden kann, um menschliche Zellen am Leben zu erhalten. Allerdings müssen wir noch nachweisen, dass DMT tatsächlich in der Zirbeldrüse des Menschen produziert wird.

Abgesehen von der DMT können wir sagen, dass die Zirbeldrüse in verschiedenen religiösen und mystischen Praktiken auf der ganzen Welt als zentral angesehen wird und eine wichtige Rolle in den Theorien von Galenos (etwa 130-200 n. Chr.) und René Descartes (1596-1650) gespielt hat (López-Muñoz \& Alamo, 2011).

Vor kurzem schlug Roney-Dougal (1989; 1991; 2001) vor, dass die Zirbeldrüse und ihre Neurochemie und Neuroanatomie für das Auftreten sogenannter «PsiPhänomene» von entscheidender Bedeutung sein können (Rogo, 1975, 1976) und weist auf die Assoziation hin, die Yogis zwischen der Zirbeldrüse und dem Ajna-Chakra herstellen. Letzteres ist das psychisch-yogische Zentrum, das die Psi-Erfahrungen bei Menschen mit erwachter Kundalini kontrolliert (Luke, 2012; Miller, 1978; Satyananda, 1972).

Als Studie der Aktivität der Zirbeldrüse zwischen korrekter und rigoroser wissenschaftlicher Methode und einem mystischen und theologischen Ansatz ist die Arbeit des italienischen Arztes Paolo Lissoni, Onkologe und Pionier bei der Auseinandersetzung mit der Psychoneuroimmunologie (PNI) besonders wertvoll und grundlegend (Lissoni, 1999). 
Laut einer kürzlichen Arbeit (Lissoni et al., 2001) war die Entdeckung der grundlegenden Rolle der Zirbeldrüse bei der Regulation des Immunsystems einer der wichtigsten Fortschritte in der Erkenntnis der neurochemischen Mediation des psychospirituellen Lebens. In Lissonis mystischer Sichtweise des Menschen, als Bild des dreieinigen Gottes in seiner biochemischen, psychologischen und spirituellen Natur, kann die Zirbeldrüse als die physische/ neuroanatomische Brücke zwischen diesen multidimensionalen Aspekten betrachtet werden (Lissoni, 1999; Lissoni et al., 2001).

Lissoni ging mit seiner stark spirituellen Sichtweise des Heilungsprozesses weiter und entdeckte, dass die verschiedenen von der Zirbeldrüse produzierten Neurohormone Krebspatienten helfen könnten, die natürliche immunbiologische Resistenz gegen das Tumorwachstum wiederherzustellen: Dies geschieht durch die Regelung des Produktionsrhythmus von vier Haupt-Indolen, die in bestimmten Zeitabständen produziert und freigesetzt werden: i) 5-Methoxytryptol (5-MLT) am Mittag; ii) 5-Methoxytryptamin (5-MT) bei Sonnenuntergang; iii) Melatonin (MLT) bei Nacht; und iv) 5-Methoxy3-Indol (5-MIAA) Essigsäure am frühen Morgen.

Diese fein abgestimmte Regulierung kann dem Patienten helfen, sich wieder mit seiner spirituellen $\mathrm{Di}$ mension zu verbinden, um von seiner Krankheit zu heilen. Laut Lissoni (1999) wird die apikale Hierarchie der Zirbeldrüse in der menschlichen Psychobiologie dadurch nachgewiesen, dass sie die einzige Struktur des menschlichen Körpers darstellt, die in der Lage ist, das Licht und den Magnetismus des Universums in eine biologische Reaktion umzuwandeln und die Funktionen des Organismus in voller Harmonie mit der Umgebung zu modulieren (ebd.).

\section{Zukünftige Entwicklungen und Perspektiven der Therapie mit psychoaktiven Substanzen}

Weitere Forschungen in dieser Richtung sind erforderlich, um die Eigenschaften von Tryptaminen wie DMT und ihre biophysikalischen Auswirkungen sowie die Auswirkungen auf die menschliche Wahrnehmung und Gehirnkommunikation besser zu verstehen.

Zusammenfassend lässt sich sagen, dass die von den Forschern gemeldeten aktuellen Erfahrungen uns sehr ermutigende Daten über den Nutzen dieser Art von Erfahrungen liefern. Die in den USA und in Europa, in Spanien und der Schweiz entwickelte Forschung folgt tendenziell Forschungsprotokollen, in denen das Set (der aktuelle Stand, aber auch die klinische und biographische Geschichte des Patienten), das Setting (Umfeld, in dem die Erfahrung stattfindet) und die Substanz (Art, Dosierung, Qualität und Quantität) eine grundlegende Rolle spielen.

Das Setting beinhaltet in der Regel einen gemütlichen Raum mit einem Sofa, das während der Sitzung benutzt wird, eine Maske zur Abdeckung der visuellen Reize, Kopfhörer, die mit einer Stereoanlage verbunden sind, und ausgebildete Fachkräfte, Psychotherapeuten und
Ärzte, die die Aufgabe haben, die Person bei der Pflegeerfahrung mit dem Einsatz der Substanz zu betreuen.

Insbesondere in Kalifornien wird derzeit ein Experiment mit dem Einsatz von MDMA bei Patienten mit PTBS (Posttraumatische Belastungsstörung) durchgeführt. Im Zusammenhang mit schweren Depressionen und Angstzuständen am Ende des Lebens werden die möglichen Vorteile der Behandlung mit Psilocybin untersucht. Die Substanz ist in Pilzen enthalten und wird seit Jahrtausenden in der uralten mexikanischen Huichol-Tradition rituell genutzt. Das Forschungsprogramm sieht die Bewertung der Symptome nach der Anwendung der Substanz und die Auswertung von Fragebögen und Durchführung von Psychotherapiesitzungen vor, um die Erfahrungen zu integrieren und für die Personen nützliche Verbindungen in der Fortsetzung ihres täglichen Lebens zu finden. Patienten berichten oft von Erfahrungen der Auflösung ihres Egos. Diese Erfahrungen sind dank neuroimaginärer Techniken mit einem starken Rückgang der Aktivität der Gehirnregionen des DMN/Default Mode Network (dt. Ruhezustandsnetzwerk), verbunden; dieser Bereich scheint während Episoden des «Grübelns und Brütens» typisch für depressive Zustände - überaktiv zu sein und spielt auch eine wichtige Rolle bei der Fähigkeit zur Selbstbeobachtung und bei der Entwicklung der Fähigkeit, die Emotionen anderer zu «lesen» und zu interpretieren.

Im Bereich der Abhängigkeiten (Sucht) sind sowohl die Nutzung pflanzlicher Substanzen wie im Falle von Ayahuasca, als auch die Behandlung mit chemischen Substanzen, wie zum Beispiel MDMA, in der Lage, alte, früher verdrängte Erinnerungen auftauchen zu lassen. Dies kann die Verarbeitung von Traumata erleichtern, die dem Problem zugrunde liegen können.

Statistische Daten sind aus offensichtlichen «praktischen» Gründen und aufgrund der Schwierigkeit, Genehmigungen zu erhalten, immer noch beschränkt verfügbar, aber die Ergebnisse haben die potenten Eigenschaften dieser uralten Mittel - in einem sicheren und kontrollierten Kontext - eindeutig hervorgehoben.

Die Herausforderungen für die Zukunft des therapeutischen Einsatzes psychoaktiver Substanzen sind nach wie vor vielfältig. Eine konkrete Möglichkeit könnte darin bestehen, den rituellen Gebrauch der Substanzen neu zu gestalten. Durch die Kombination von Vergangenheit und Gegenwart, von uraltem Wissen und modernster Wissenschaft im Dienst der Behandlung.

\section{Literatur}

Ahearne-Kroll, S. P. (2014). The afterlife of a dream and the ritual system of the Epidaurian Asklepieion. Archiv für Religionsgeschichte, 15(1), 35-51.

Al-Balhan, E. M., Khabbache, H., Watfa, A., Re, T.S., Zerbetto, R. \& Bragazzi, N. L. (Hrsg.). (2018). Psychometric Evaluation of the arabic version of the nomophobia questionnaire: confirmatory and exploratory factor analysis - implication from a pilot study in Kuwait among university students. Psychology Research and Behavioural Management, 11, 471-482.

Aldridge, D. (2000). Spirituality, Healing and Medicine: Return to the Silence. London: Jessica Kingsley. 
Bragazzi, N.L., Khabbache, H., Perduca, M., Neri, B., Firenzuoli, F., Penazzi, G., Simões, M., Zerbetto, R. \& Re, T.S. (2018a). Para-Psychology, N,N-Dimethyltryptamine and the Pineal Gland. Cosmos and History: The Journal of Natural and Social Philosophy, 12(2), 228-238.

Bragazzi, N. L., Khabbache, H., Vecchio, I., Martini, M., Perduca, M., Zerbetto, R. \& Re, T. S. (2018b). Ancient Shamanism and Modern Psychotherapy: From Anthropology to Evidence-Based Psychedelic Medicine. Cosmos and History: The Journal of Natural and Social Philosophy, 14(1), 142-152.

Bragazzi, N.L., Khabbache, H., Vecchio, I., Martini, M., Zerbetto, R. \& Re, T.S. (2018c). Neurotheology of Islam and higher consciousness states. Cosmos and History: The Journal of Natural and Social Philosophy, 14(2), 315-321.

Bragazzi, N.L., Re, T.S., Zerbetto, R., Firenzuoli, F. \& Gensini, G. F. (2018d). Umbrella review of medicinal cannabis. Phytomedicine Journal, 2018 (submitted).

Bulkeley, K. (2008). Dreaming in the World's Religions: A Comparative History. New York: University Press.

Drury, N. (2011). Stealing Fire from Heaven: The Rise of Modern Western Magic. Oxford: University Press.

Edelstein, E. J. \& Edelstein, L. (1945). Asclepius. Collection and Interpretation of the Testimonies. Baltimore: John Hopkins Press.

Firenzuoli, F., Maggini, V., Re, T.S., Sivelli, F., Gallo, E. \& Gensini, G.F. (2018). Clinical phytotherapy and herbal pharmacovigilance [eBook; www.sci9.org/ebooks].

Firenzuoli, F., Sivelli, F., Maggini, V., Re, T.S., Gallo, E. \& Gensini, G.F. (2018). Medicinal Cannabis in Italy: Current Events and Perspectives [eBook; www.sci9.org/ebooks].

Gesler, W.M. (1993). Therapeutic Landscapes: Theory and a Case Study of Epidauros, Greece. Environment and Planning D: Society and Space,11(2), 171-189.

Gesler, W.M. (2003). Healing Places. Rowman \& Littlefield.

Gulluni, N., Re, T.S., Loiacono, I., Lanzo, G., Gori, L., Macchi, C., Epifani, F., Bragazzi, N. L. \& Firenzuoli, F. (2018). Cannabis Essential Oil: A Preliminary Study for the Evaluation of the Brain Effects. Evidence-Based Complementary and Alternative Medicine, 2018. https://doi.org/10.1155/2018/1709182

Hart, G. D. (2000). Asclepius: The God of Medicine. London: Royal Society of Medicine Press.

Hoot, D. R. (2014). The Sanctuary of Asclepius at Epidaurus in Roman Times. Gainesville: University Press of Florida.

Lissoni, P. (1999). The pineal gland as a central regulator of cytokine network. Neuro Endocrinol Letters, 20(6), 343-349.

Lissoni, P., Cangemi, P., Pirato, D., Roselli, M. G., Rovelli, F., Brivio, F., Malugani, F., Maestroni, G. J., Conti, A., Laudon, M., Malysheva, O. \& Giani, L. (2001). A review on cancer-psychospiritual status interactions. Neuro Endocrinology Letters, 22(3), 175-80.

López-Muñoz, F. \& Alamo, C. (2011). Cartesian theories on the passions, the pineal gland and the pathogenesis of affective disorders: an early forerunner. Psychological Medicine 41(3), 449-51.

Luke, D. (2011). Discarnate entities and dimethyltryptamine (DMT): Psychopharmacology, phenomenology and ontology. Journal of the Society for Psychical Research, 75, 26-42.

Luke, D. (2012). Psychoactive substances and paranormal phenomena: A comprehensive review. International Journal of Transpersonal Studies, 31(1), 97-156.

Malatesta, M. (Hrsg.). (2015). Doctors and Patients: History, Representation, Communication from Antiquity to the Present. San Francisco: University of California Medical Humanities Press.

McKenna, T. (1992). Food of the Gods. New York: Bantam.

Meier, C. A. (2012). Healing Dream and Ritual: Ancient Incubation and Modern Psychotherapy. Einsiedeln: Daimon.

Perriam, G. (2015). Sacred spaces, healing places: therapeutic landscapes of spiritual significance. Journal of Medical Humanities, 36(1), 19-33.

Petsalis-Diomidis, A. (2010). Truly Beyond Wonders: Aelius Aristides and the Cult of Asklepios. Oxford: University Press.
Pettis, J.B. (2015). The Sleeper's Dream: Asclepius Ritual and Early Christian Discourse. Piscataway: Gorgias Press.

Re, T.S., Palma, J., Martins, J.E. \& Simões, M. (2016). Transcultural perspective on consciousness: Traditional use of ayahuasca in psychotherapy in the 21st Century in Western World. Cosmos and History: The Journal of Natural and Social Philosophy, 12(2), 237-249.

Re, T.S. \& Ventura, C. (2015). Transcultural Perspective on Consciousness: a bridge between Anthropology, Medicine and Physics. Cosmos and History: The Journal of Natural and Social Philosophy, 11(2), 228-241.

Renberg, G.H. (2017). Where Dreams May Come Incubation Sanctuaries in the Greco-Roman World (Vol. I). Leiden and Boston: Brill.

Roney-Dougal, S.M. (1989). Recent findings relating to the possible role of the pineal gland in affecting psychic ability. Journal of the Society for Psychical Research, 55, 313-328.

Roney-Dougal, S. M. (1991). Where Science and Magic Meet. Shaftesbury, Dorset: Element Books.

Roney-Dougal, S.M. (2001). Walking between the worlds: Links between Psi, Psychedelics, Shamanism and Psychosis. Glastonbury: Psi Research Centre.

Walton, A. (1894). The cult of Asklepios. Boston: Ginn \& Company. Williams, A. (1998). Therapeutic Landscapes in Holistic Medicine. Social Science and Medicine. 46(9), 1193-1203.

Winkelman, M.J. (1992). Shamans, Priests and Witches. A Cross-Cultural Study of Magico-Religious Practitioners. Tempe: Arizona State University.

\section{From the Rite of Incubation to Psychotherapy. A transcultural perspective}

Abstract: Originally, in the West, medicine, which concerned the care of body, mind and soul, was a divinatory art. In the course of time, the term «divination» lost it's original meaning in comparison to antiquity and especially to the archaic Greek world, where it had an enormous value. The art of Asclepius, like that of his father Apollo, is a divine art that requires a healing ritual. In this context, it should be remembered that historical anthropological research in many cultures of the shamanic type (South America, Siberia, India) records a very ancient therapeutic use of "master plants» or plants which, because of their characteristics, make it possible to reach «extraordinary states of consciousness». These states of consciousness can arise through various practices such as meditation, holotropic breathing, yoga, sensory deprivation or even spontaneously as with Christian mystics. The current experiences described by the researchers provide us with very encouraging data on the benefits of this kind of knowledge. Research developed in the USA and in Europe, Spain and Switzerland tends to follow research protocols in which the set (the current status, but also the clinical and biographical history of the patient), the setting (the environment in which the experience takes place) and the substance (type, dosage, quality and quantity) play a fundamental role. The challenges for the future in the psychotherapeutic field, through the therapeutic use of psychoactive substances, remain diverse, and a real possibility could be to revitalize the ritual use of substances by combining past and present, ancient knowledge and modern science in the service of care.

Key Words: Asklepios (Aesculap), Epidauros, sapiental medicine ( «wisdom medicine»), temple medicine, incubation sanctuary, master plants, shamanism 


\section{Die Autorin}

Tania Re, diplomiert in klinischer und Gemeindepsychologie, ist Anthropologin mit den Schwerpunkten Medizinethnologie (Medical Anthropology) und Ethnomedizin; sie ist als ergänzende Therapeutin und Psychologin in der Schweiz tätig. Als Gründungsmitglied der Cattedra Unesco «Antropologia della salute, Biosfera e sistemi di cura» (UNESCOLehrstuhl für «Gesundheit, Anthropologie, Biosphäre und Behandlungssysteme») an der Universität Genua (IT) und wissenschaftliche Mitarbeiterin am Regionalen Zentrum für Phytotherapie der Toskana (CERFIT) - Careggi University Hospital Florenz (IT) ist sie am Studium der Behandlungssysteme interessiert, die aus traditionellen Heilpraktiken stammen und Geist, Körper und Seele verknüpfen.

\section{Kontakt}

E-Mail: tania.re77@gmail.com 